\title{
CONCEPTS
}

\section{Acute Hamstring Muscle Tears in Climbers-Current Rehabilitation Concepts}

\author{
Uzo Dimma Ehiogu, MSc, BSc, BSc ${ }^{1,2,3}$; Gareth Stephens, MSc, BSc ${ }^{1}$; Gareth Jones, $\mathrm{PhD}, \mathrm{MSc}^{3}$; \\ Volker Schöffl, MD, MHBA, PhD ${ }^{3,4,5,6}$ \\ ${ }^{1}$ Birmingham Royal Orthopaedic Hospital, Research and Training Department, Birmingham, United Kingdom; ${ }^{2}$ Birmingham Medical School, College of \\ Medical and Dental Sciences, University of Birmingham, Birmingham, United Kingdom; ${ }^{3}$ School of Clinical and Applied Sciences, Leeds Beckett Univer- \\ sity, Leeds, United Kingdom; ${ }^{4}$ Department of Orthopedic and Trauma Surgery, Klinikum Bamberg, Friedrich Alexander University Erlangen-Nuremberg, \\ Erlangen, Germany; ${ }^{5}$ Section of Sports Medicine, Department of Orthopedic Surgery, Klinikum Bamberg, Germany; ${ }^{6}$ Department of Emergency \\ Medicine, University of Colorado School of Medicine, Aurora, $\mathrm{CO}$
}

\begin{abstract}
Acute hamstring injuries are often caused by the heel hook technique. This technique is unique to climbing and causes injury to muscular and inert tissues of the posterior thigh. The heel hook is used by climbers during strenuous ascent on overhanging walls and when crossing difficult terrain. The technique reduces the amount of upper body strength required during strenuous climbing because the climber's center of mass is retained within the base of support. The heel hook is stressful collectively for the hamstring muscle group and musculotendinous junction. Depending on injury severity, both conservative and surgical methods exist for the management of hamstring injuries. Contemporary approaches to rehabilitation primarily advocate the use of eccentric muscle strengthening strategies because of high rates of elongation stress associated with sprinting and team sports. However, there is reason to doubt whether this alone is sufficient to rehabilitate the climbing athlete in light of the high degree of concentric muscle strength required in the heel hook maneuver. This review examines the contemporary rehabilitation and strength and conditioning literature in relation to the management of acute hamstring musculotendinous injuries for the climbing athlete. The review provides a comprehensive approach for the rehabilitation and athletic preparation of the climbing athlete from the initial injury to full return to sports participation.
\end{abstract}

Keywords: rehabilitation, heel hook, hamstring injury, climbing injury

\section{Introduction}

A wide spectrum of hamstring-related injuries is reported in the literature. ${ }^{1,2}$ This includes complete and partial proximal hamstring tendon avulsions, ischial apophyseal avulsions, proximal hamstring tendinopathy, referred posterior thigh pain, and hamstring tear. ${ }^{2}$ Muscle injuries are very common in sports. ${ }^{3}$ An acute muscle tear is an injury affecting the structural integrity of the muscle fibers/bundles, leading to loss of continuity and contractile properties of

Corresponding author: Uzo Dimma Ehiogu, MSc, BSc, BSc, University of Birmingham, Birmingham Royal Orthopedic Hospital, Research and Training Department, Birmingham Medical School, College of Medical and Dental Sciences, The Woodlands, Bristol Road, Birmingham B31 2AP, United Kingdom; e-mail: uzo.ehiogu@nhs.net.

Submitted for publication August 2019.

Accepted for publication July 2020. the muscle. ${ }^{4}$ Acute hamstring tears are the most common hamstring-related injury resulting in loss of time from sports and training for athletes. ${ }^{1}$ Acute hamstring tears have increased susceptibility to recurrent injury. It has been suggested that up to one-third of hamstring strains will recur within the first 2 wk of return to sports. ${ }^{5}$ This high recurrence rate may be suggestive of inadequate rehabilitation and premature return to sports.

The prevalence of hamstring injury is high in sports that require kicking or highly skilled movements, such as soccer, ${ }^{6}$ Australian-rules football, ${ }^{7}$ Gaelic football, ${ }^{8}$ and sprinting. ${ }^{9}$ Acute hamstring muscle strains have been found to be more common in sports that involve significant muscle lengthening and elongation while under active tension. ${ }^{10}$ This injury mechanism is a common factor associated with high-speed sprinting and is suggested to account for the majority of these injuries in team sports. The 
terminal swing phase in sprinting has been proposed to be a cause of injury for the hamstring muscles because of the rapid eccentric muscle lengthening required to decelerate the tibia in preparation for ground contact. ${ }^{11}$ The degree of elongation of the biarticular hamstring muscles is substantial in the terminal swing phase because of the magnitude of hip flexion and knee extension range of motion required. ${ }^{12}$ The biarticular muscles (long head of biceps femoris, semimembranosus, and semitendinosus) have been reported to be elongated 12,10 , and $9 \%$, respectively, beyond their normal upright length. ${ }^{13}$

Furthermore, peak hamstring muscle force and eccentric work has been reported during this phase with increasing speed of limb motion. ${ }^{14}$ This may explain the preference for eccentric strength training in the management of hamstring strains in the literature and clinical practice. Eccentric training has been shown to increase fascial length, which has been attributed to an increase in sarcomeres in series within the muscle. ${ }^{15}$ This may cause a shift in the length-tension relationship of the muscle, allowing the production of peak force in a lengthened position such as the terminal swing phase. ${ }^{16}$ However, we believe acute hamstring injuries in climbers favor a different mechanism of injury when compared to sprinting. The mechanism of injury is the heel hook technique, which uses concentric muscle work and to a lesser degree an isometric muscle action. This, we believe, makes it difficult to extrapolate eccentric training modes to hamstring rehabilitation because of training mode specificity.

The heel hook technique has been associated with injuries to the lower extremities. Injuries range from hamstring avulsion injuries, posterior cruciate ligament injuries, and posterior meniscal injuries to hamstring muscle partial thickness tears. ${ }^{17}$ Depending on the severity and nature of the injury, both conservative and surgical methods exist for the management of hamstring injuries. ${ }^{2}$ Soft tissue rehabilitation is a specialized area of sports medicine and physiotherapy concerned with the restoration of appropriate soft tissue and neurodynamic function.

Conservative management and rehabilitation of the intramuscular tendon and adjacent muscle fibers after a heel hook-related injury have not been previously reported. Current guidance on the rehabilitation of hamstring injuries in athletic populations tends to focus on eccentric muscle rehabilitation. ${ }^{10}$ However, the heel hook technique requires significant concentric hamstring muscle strength to propel the climber and isometric strength to maintain a static position. Therefore, it is questionable whether current eccentric loading programs alone are appropriate to rehabilitate the climbing athlete. Therefore, the aim of this paper is to present a rehabilitation model for the climbing athlete with an acute hamstring muscle injury who needs to perform the heel hook technique. A second objective is to provide an evidence-based approach for reconditioning and return to sports for the climbing athlete.

\section{Hamstring Muscle Group}

\section{ANATOMY}

The hamstring muscle group consists of 3 muscles: the biceps femoris (long and short heads), the semimembranosus, and semitendinosus. ${ }^{18}$ The long head of the biceps femoris, the semimembranosus, and the semitendinosus all form a common tendon of origin originating from the ischial tuberosity. The short head originates medial to the linea aspera on the posterior distal femur. The long head of the biceps femoris takes its distal attachment at the fibular head and lateral tibia. The short head of the biceps femoris attaches to the tendon of the long head of the biceps femoris, blending with the fascial and tendinous insertions of the posterolateral capsule and the iliotibial tract. The semitendinosus blends and joins with the sartorius and gracilis tendons, forming the pes anserinus distal to the medial collateral ligament. The long head of the biceps femoris, the semimembranosus, and the semitendinosus are neurologically innervated by the tibial portion of the sciatic nerve and the short head of the biceps femoris by the peroneal portion of the sciatic nerve. The biceps femoris is a flexor of the knee and also externally rotates the knee. ${ }^{19}$

\section{FUNCTION}

The hamstring muscle group functions to flex and to internally and externally rotate the knee and extend the hip. The semimembranosus and semitendinosus, in addition to flexing the knee, also internally rotate the knee. The biceps femoris is a flexor of the knee and also externally rotates the knee. The long head of the biceps femoris and semimembranosus and semitendinosus act as primary hip extensors alongside the gluteus maximus. ${ }^{18}$

\section{Diagnosis}

The clinical diagnosis can be made by a physician or physical therapist, depending on qualifications and expertise. Patients often present with a history of acute pain in the posterior thigh or buttock correlating with an antalgic gait and reduced muscle performance in the hamstring group. ${ }^{2}$ Physical examination may reveal point tenderness on palpation of the injured area and ecchymosis, which may or may not track distally. Passive movement testing of the knee into extension and hip flexion is often symptomatic because of the tensile forces involved. Manual muscle testing often reproduces pain and weakness of the hamstring muscles. ${ }^{20}$ Hamstring injuries are generally graded in respect to their severity clinically (Table 1). 
Table 1. Hamstring injury severity classification system (adapted from Ahmad et al 2013²)

\begin{tabular}{ll}
\hline grade 1 (mild) & Overstretching with minimal loss of structural integrity of the muscle-tendon unit \\
grade 2 (moderate) & Partial or incomplete tearing of the muscle-tendon unit \\
grade 3 (severe) & Complete rupture of muscle-tendon unit \\
\hline
\end{tabular}

After the clinical examination, radiologic investigation may be required to confirm the clinical diagnosis. Plain radiography is indicated in posterior thigh injuries if an avulsion fracture of the ischial tuberosity is suspected. ${ }^{2}$ Magnetic resonance imaging and ultrasonography are radiologic modalities of choice for soft tissue injuries. However, where there is diagnostic uncertainty with regard to the anatomic site and severity of injury, magnetic resonance imaging has been shown to have greater sensitivity, identifying subtle fluid changes and anatomic location and defining the injury classification. ${ }^{21,22}$

\section{Heel Hook Technique}

The heel hook technique is unique to climbing athletes (Figure 1). The technique involves a pulling motion, creating friction between the climber's heel and the rock. The technique has multiple uses in climbing: It can be used to stabilize the climber to facilitate upper body movement on difficult terrain, which can reduce load through the upper body and conserve energy for longer or difficult climbs, and it can generate upward momentum of the climber's center of mass in its own right. ${ }^{17}$

The popularity of indoor climbing has changed the nature of climbing and hence, we believe, the prevalence and incidence of injuries. The incidence of injuries affecting the lower limbs in sport climbing and bouldering has grown steadily in recent years. ${ }^{23}$ The change in injury

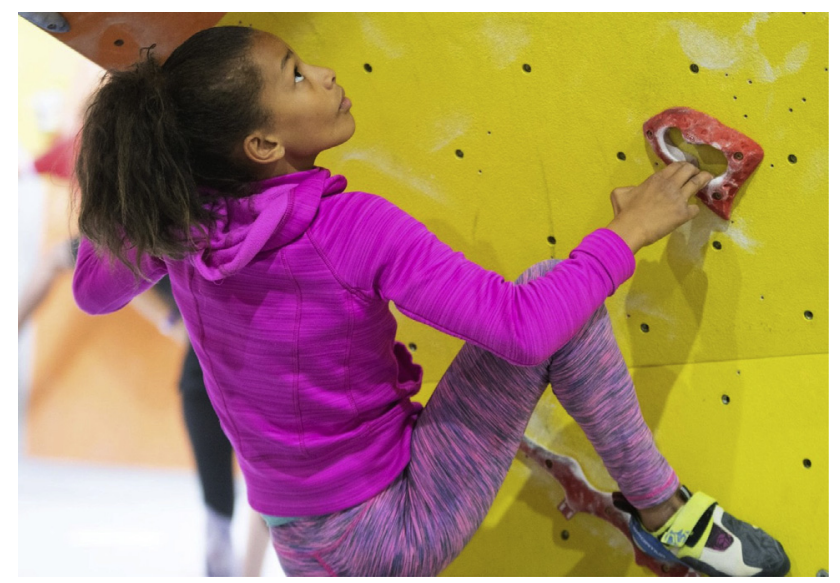

Figure 1. A climber performing a heel hook on an artificial climbing wall. distribution is not known for certain, but increased participation in bouldering may have affected the incidence. ${ }^{17,23}$ Bouldering, a derivative of rock climbing, has seen rising participation levels in the past decade. ${ }^{24} \mathrm{~A}$ bouldering problem is a sequence of difficult moves without the use of ropes or gear for protection. The heel hook technique is used extensively in bouldering to reduce the force and power requirements of the upper body musculature.

The physiologic demands of this style of climbing are very different from other forms of rock climbing. ${ }^{25}$ The demands of competition bouldering are characterized by significant utilization of anaerobic energy metabolism to facilitate successful performance. ${ }^{26}$ The physiologic profile of the bouldering specialist is one of marked reliance on maximal muscle strength and force expression. ${ }^{27}$ This style of climbing is typified by training practices that involve the skillful expression of force in a climbing environment and high rates of force development.

The kinematic or biomechanical movement profile associated with bouldering imposes a high degree of both upper and lower limb involvement to ensure strong attraction to the rock. The lower limb in particular is characterized by manipulation of the heel, knee, and hip articulations to create friction on the rock to generate propulsive force to transition from one body position to the next. This reliance on the lower limb to generate high force production may contribute to the occurrence of heel hook-related injuries in bouldering. The heel hook technique has been associated with injuries to the lower extremity ranging from hamstring avulsion injuries, posterior cruciate ligament injuries, and posterior meniscal injuries to hamstring muscle partial thickness tears. ${ }^{17}$ Depending on the severity and nature of the injury, both conservative and surgical methods exist for the management of hamstring injuries. ${ }^{2}$

\section{Rehabilitation and Physical Preparation Considerations}

Soft tissue rehabilitation is a specialized area of sports medicine and physiotherapy, concerned with the restoration of appropriate soft tissue and neurodynamic function. Conservative management and rehabilitation of the intramuscular tendon and adjacent muscle fibers after a heel hook-related injury have not been previously reported. Current guidance on the rehabilitation of hamstring injuries 
in athletic populations tends to focus on eccentric muscle rehabilitation. ${ }^{10}$ However, the heel hook technique requires significant concentric hamstring muscle activity to propel the climber. Therefore, it is questionable whether current eccentric loading programs are appropriate to rehabilitate the climbing athlete. The aim of this paper is to present a rehabilitation model for the climbing athlete with a hamstring muscle injury who needs to perform the heel hook technique. A second objective is to provide an evidencebased approach for reconditioning and return to sports among climbing athletes (Table 2).

\section{THE NEEDS ANALYSIS}

An analytical analysis of the sport and specific movement pattern involved in the mechanism of injury is an important component in developing a comprehensive rehabilitation plan for the injured climbing athlete. The analysis should consider the physiologic biomechanical profile and the athlete's predisposing factors for the initial injury. It is also important to consider the athlete's social and psychological status as a part of a biopsychosocial framework. However, a discussion of the psychological and social determinates of rehabilitation is beyond the scope of this review.

\section{THE PHYSIOLOGICAL PROFILE}

The physiological determinants of an activity are determined by the nature of the metabolic energy systems used during its performance. The magnitude and duration of the forces required by the muscle group to perform the activity largely determine the metabolic demand. Bouldering is heavily dependent on the alactic and fast glycolytic systems because of the short durations of activity. ${ }^{26}$ The heel hook technique is used to either hold a proportion of the climber's body weight or to propel the climber's center of mass from one position to another. ${ }^{28}$ The short duration of this activity is reliant on maximal strength expression and explosiveness. ${ }^{18}$

Deconditioning after injury is a concern for recreational and competitive climbers. It is therefore important for healthcare professionals to appreciate the physical demands associated with the sport. This will assist the clinician to select conditioning activities that help to maintain climbing performance without affecting the recovering injury. This analysis of the physical attributes associated with performance requires an understanding of the underlying physiologic mechanisms pertinent to physical capacity. For example, the mechanisms that contribute to accelerating the upper body vertically to latch on to a hold are knee and hip extension strength, ${ }^{29}$ trunk stiffness ${ }^{30}$ forearm flexor muscle cross sectional area, ${ }^{31}$ and the rate of force development of the finger flexors and lower body extensors. Furthermore, it has been shown that high levels of finger strength can differentiate elite climbers from their nonelite counterparts. ${ }^{31}$ Static trunk strength, upper limb strength, and power are moderately associated with elite

Table 2. The rehabilitation/reconditioning continuum (adapted from Comfort et al 2009 ${ }^{59}$ )

Initial treatment

Restoring range of motion

Phase 1: Initial strengthening

Phase 2: Low velocity strengthening

Phase 3: High load strengthening and functional reconditioning

Return to climbing-specific considerations
Protection from further injury, rest, cryotherapy, compression, and elevation are generally accepted for muscle-tendon unit injuries in the acute phase ${ }^{3}$

- Stretching to increase tensile load without symptom reproduction on a daily basis, 3 to 4 sets, 30 to $60 \mathrm{~s}$ per stretch $4 \times$ per day ${ }^{4}$

- Sciatic nerve mobilizations performed daily using either sliders or longitudinal tensioners-dosage is variable ${ }^{5}$

- Isometric hamstring specific strengthening exercises in multiple ranges of motion ${ }^{6}$

- Specific hamstring exercises (eg, moderate-weight resistive bands, leg curl machines, and double-leg stiff leg lifting in a pain protected range of motion) ${ }^{6-9}$

- Begin regional upper-quadrant sports-specific muscle conditioning, including finger strengthening ${ }^{10}$

Low velocity concentric full range of motion hamstring-specific exercises with increasing resistive load using machines and free weights (eg, heavy elastic bands, knee curl machines, ${ }^{6-9}$ and deadlifts ${ }^{7}$ )

- High resistive loads in both outer and inner range of motion ${ }^{6,11,12}$

- Unilateral and single leg modes (eg, single-leg stiff leg dead lifting, kettle bell Romanian dead lifting, single-leg knee curls with and without external tibial rotation) $)^{13,46}$

- Gradually increase the speed of movement to optimize RFD-incorporate ballistic training as required ${ }^{14}$

Progressive development of sports-specific heel hook strength by a reduction in assistance from the upper limb (eg, heel hook with significant upper limb assistance [jug hold], then half crimp to full crimp, vertical then to overhanging rock or wall) 
climbing performance ${ }^{32,33}$; therefore, determining the performance-limiting factors offers a high degree of training specificity for the injured climber.

\section{BIOMECHANICAL CONSIDERATIONS}

An in-depth understanding of the biomechanical demands of climbing and the heel hook technique is important for treatment and management planning. Kinematics refers to the spatial and temporal characterizations of the task without consideration of the forces involved. An understanding of the heel hook angular kinematics and planes of motion is important for selecting therapeutic exercise for rehabilitation. The degree of variability and the complexity of the movement pattern can be reduced by establishing the dominant technical model for heel hooking. Qualitative analysis of this activity suggests that the movement is initiated with hip and knee flexion and that movement can occur from 10 to 90 degrees of knee flexion. ${ }^{28}$ Tibial rotation ("predominately lateral rotation"), when combined with knee flexion, may create a mechanical advantage for the posterior lateral hamstring muscles. ${ }^{17}$ The superficial planter flexor group (gastrocnemius and soleus) is also functionally relevant to the heel hook technique. The gastrocnemius and soleus assist the hamstring group by providing powerful plantar flexion torque. ${ }^{34}$ This may increase frictional forces between the climber's heel and the rock. This is suggestive of the need to rehabilitate hamstring muscle function in both outer and inner range of motion, with and without tibia rotation and plantar flexion (Figures 2-5).

The kinetic analysis examines the forces and types of muscle actions involved. The forces involved in the heel hook technique have not been evaluated previously in the literature. However, observational analysis of the kinematics suggests dominance of concentric hamstring muscle activity to propel the climber from one position to the next. There is also an isometric hamstring muscle component when the climber is stationary and, to a lesser degree, some eccentric muscle activity. This analysis of muscle

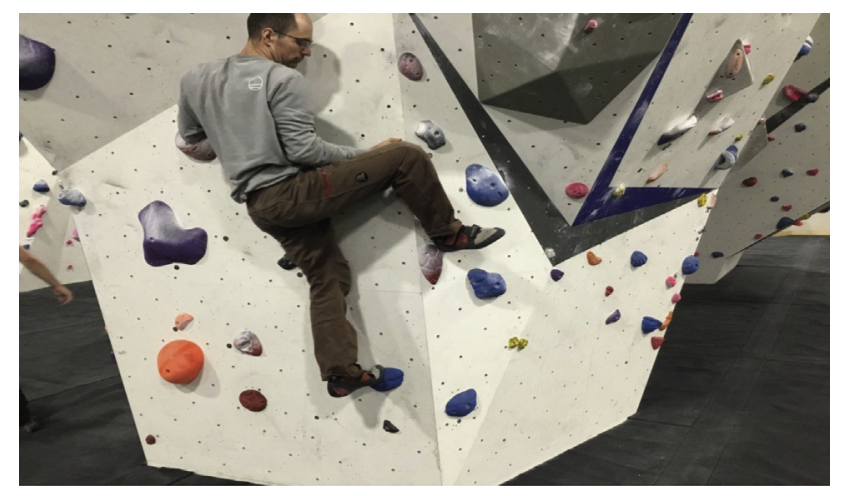

Figure 2. Initiation of the high heel hook technique.

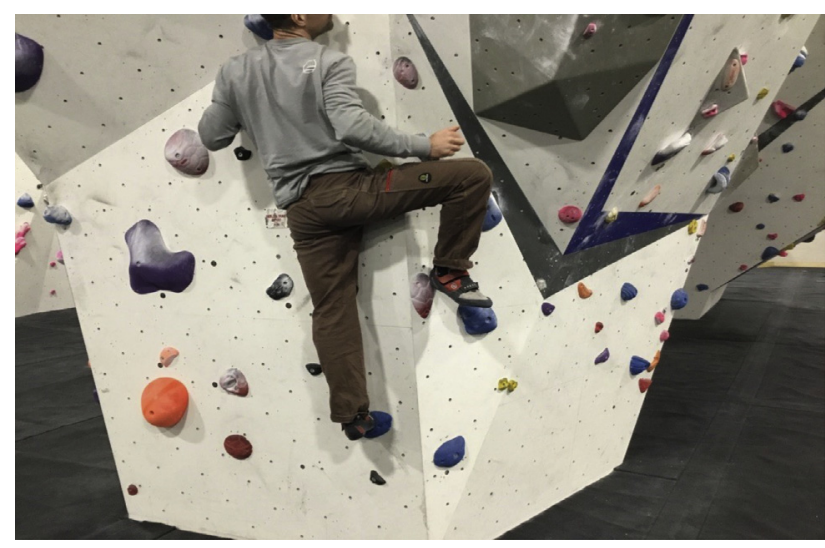

Figure 3. Concentric activation of the hamstring musculature pulling the hip toward the knee.

function is important because there is a significant amount of literature on hamstring muscle rehabilitation in running ${ }^{16,35-37}$ and sprinting-based sports that the practitioner may be tempted to apply to the climbing athlete. However, this literature is primarily concerned with eccentric strengthening of the hamstring musculature. The theoretical model evident in this analysis of muscle function is primarily concerned with deceleration of the tibia during sprinting and running. ${ }^{35,38,39}$ For the climber, the mechanism of injury and subsequent muscle action favors acceleration of the tibia by concentric muscle action. ${ }^{17,28}$ Therefore, eccentric loading research in sprinting and team sports populations may be less relevant to the sport of climbing, underscoring the importance of a sports-specific needs analysis.

Additionally, because of the close anatomic and biomechanical relationship between the hamstring and the core musculature, it would seem prudent to understand the role of the trunk in this activity. ${ }^{40}$ Although no primary data

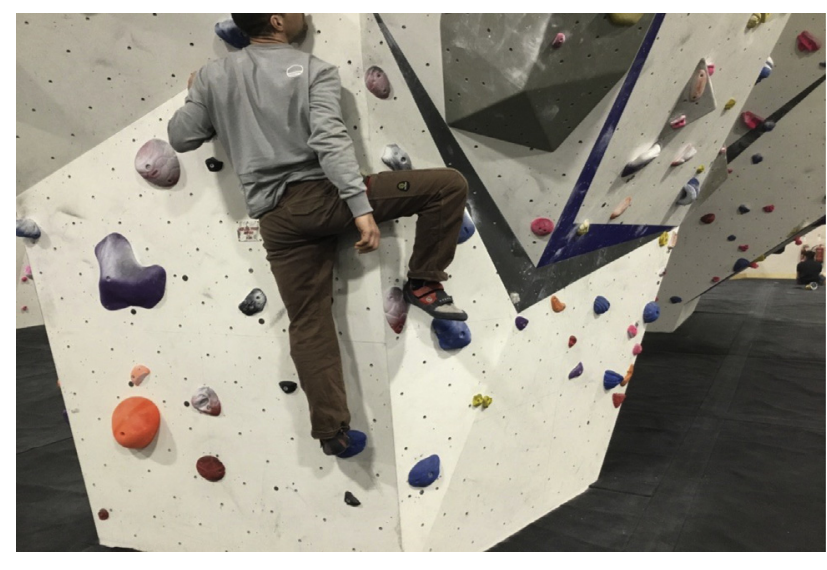

Figure 4. Isometric activation of the hamstring assisting the climber's stationary position. 


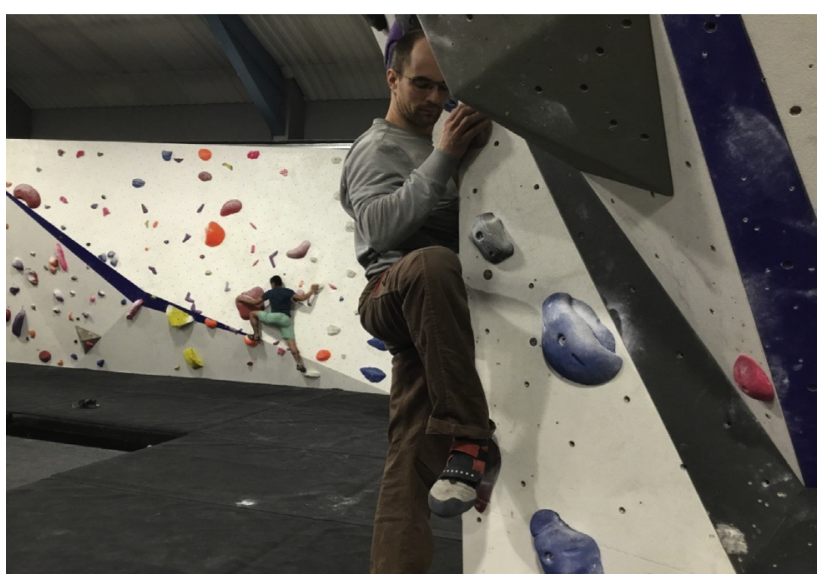

Figure 5. Inner range knee flexion with associated tibial external rotation.

are available on the trunk muscle forces involved in the heel hook technique, it is generally accepted that both static and dynamic trunk strength are important for sports performance. ${ }^{41}$ Indeed, maximal isometric trunk strength while pulling via the heel in a static holding pattern would seem to be performance enhancing via effective transmission of force. Moreover, when transitioning from one position to another, concentric maximal trunk strength and rate of force development may also be important to the climbing athlete.

\section{Conservative Management of the Musculotendinous Junction Unit}

PHASE 1: CONTROL OF PAIN AND THE INITIAL STRENGTHENING PHASE

\section{Control pain}

The initial injury is usually associated with a significant amount of localized pain over the site of injury. The aim in the initial phase is to control pain and to allow the timely resumption of controlled tensile forces through the injured tissues. Control of pain may be far more important than control of inflammation, as was previously thought. ${ }^{42}$ Inflammation control with nonsteroidal anti-inflammatory drugs in the initial stages of a muscle injury should be used with caution because growing evidence suggests that use of these drugs can retard the healing process and affect the quality of collagen. ${ }^{43-45}$ Therefore, the use of nonsteroidal anti-inflammatory drugs with this type of injury needs to be carefully considered in regard to its timing, dosage, and overriding rationale.

\section{Prevent excessive scar tissue formation}

To prevent excessive and disorganized deposition of scar tissues, it is important to begin the application of tensile forces through the injured tissues early. This will promote appropriate remodeling of tissues along the target tissue's lines of stress. Activities such as controlled mobilization and stretching exercises, which empower patients to take an active role in their rehabilitation, are preferable. ${ }^{5}$ At this stage, it is also important to ensure that the patient has appropriate sciatic nerve mobility. Mechanical sensitivity of the sciatic nerve has been associated with persistent posterior thigh pain and been implicated as a cause of reduced hip and knee range of motion. ${ }^{46,47}$ There is also a place for adjunctive passive therapies that facilitate soft tissue loading, such as manual therapies. However, in this early phase of the injury, the practitioner must always remain vigilant of exacerbating the patient's symptoms and delaying healing. We believe that the response of the patient to manual and tensile loading can be pragmatically appreciated by the degree of irritability experienced by the patient after each procedure. Irritability is the degree to which a patient's symptoms settle after exacerbation and can be used as a criterion to guide tensile loading in the early stages.

\section{Establish isometric pain-free baseline}

Isometric and concentric strength baselines should be established as soon as is practically possible to understand the practical dosage of tensile load permissible to the athlete. It is common to start with isometric-based strengthening exercises because of their ease of application. ${ }^{1}$ However, we believe it is more prudent to apply concentric-based loading through a pain-free range of motion than to delay rehabilitation by prestaging with isometrics. It is also important to understand how the constraints of a particular exercise modality will influence morphologic and mechanical adaptations on the muscular tendinous unit. Neuromechanical changes in motor unit recruitment are paramount to reduce the deleterious effects of muscle inhibition, and the abolishment of pain has been shown to reduce muscle inhibition. ${ }^{38}$ Appropriate stimulation of contractile tissues is prudent to facilitate intramuscular coordination. ${ }^{48}$ Novel therapies such as blood flow restriction training have shown some efficacy in the management of musculoskeletal injuries. ${ }^{49}$ This therapy has been shown to increase muscle strength and endurance when combined with lowintensity resistive exercise (below $20-30 \%$ of repetition maximum) compared to high-intensity resistive exercise. The mechanistic cause is still unknown; however, it has been hypothesized that vascular restriction causes the premature recruitment of high-threshold motor units and protein synthesis due to oxygen restriction and intermuscular metabolite accumulation. ${ }^{50}$ However, where and how the clinician starts the loading regime will largely be influenced by the severity of the initial injury, equipment availability, and the stage of tissue healing. 
Maintain regional upper-quadrant sports-specific muscle performance

In this early phase, it is recommended that the clinician and athlete take a proactive approach to maintain muscle performance in the sports-specific muscle groups closely associated with climbing. There is evidence to suggest that forearm muscle strength is a performance differentiator between elite and novice climbers. ${ }^{33}$ Therefore, derivative training to improve and or maintain finger strength capacities is recommended for the injured climber. ${ }^{25,31}$ Training of the trunk and lumbopelvic region at this stage should be avoided or at least approached with caution because of the close anatomic and biomechanical relationship associated with the hamstring muscles. ${ }^{51}$

\section{PHASE 2: THE LOW-VELOCITY STRENGTHENING PHASE}

In this phase, pain should no longer be the dominant feature of the athlete's presentation. It is important to increase the loading stimulus on the injured tissues. The principle of overload is a critical component because this, alongside other factors, will dictate the morphologic and mechanical adaptations sought by the clinician. ${ }^{52,53}$ The emphasis should still be on the achievement of appropriate intramuscular and intermuscular coordination. ${ }^{54}$ Loading of the tissues in isolated concentric strength training should take precedence.

Some evidence suggests strength training is mode and velocity specific, and eccentric training may not restore concentric deficits in neuromuscular function. ${ }^{55-57}$ Isolated loading of the hamstring muscle group is designed to induce changes in the intramuscular physiology of the muscle. The literature suggests that acute changes in motor unit recruitment, rate coding (firing rates), and the sequencing of motor unit recruitment at the muscle fiber level can facilitate positive adaptations in strength without an appreciable change in muscle morphology. ${ }^{58,59}$ This is an acute adaptation, in contrast to long-term adaptations in muscle cross-sectional area. Long-term adaptations are a precursor for maximizing the force-generating capacity of muscle. ${ }^{60}$ This has been shown to provide a platform for additional gains in strength and explosiveness across multiple sports. ${ }^{61}$ Additionally, heavy resistance training has been shown to provide positive morphologic adaptations in both contractile and noncontractile tissues, which is beneficial in a tissue injury model. ${ }^{60}$

The method of loading can include both machine-based and free weight-based activities. The machine-based modalities are preferable at this stage because of their ability to preferentially develop isolated overload in the target tissues. ${ }^{16}$ This has been suggested to provide a more measurable overload because of the low requirements for body stabilization and skill. ${ }^{59}$ There is some debate in the literature about the acute patterns of hamstring muscle activation during different exercises. ${ }^{62,63}$ Studies using functional magnetic resonance imaging have consistently shown preferential recruitment of the long head of the biceps femoris and semimembranosus activation during hip extension-derived movements (eg, the stiff leg deadlift). ${ }^{38}$ Knee flexion-based exercises (eg, leg curls) have shown preferential activation of the short head of the biceps and increased semimembranosus recruitment. ${ }^{1,64}$ Therefore, during the initial stages of the injury, an accurate diagnosis will aid in customized exercise prescription and prevent inappropriate loading of the injured hamstring muscle. This will allow the clinician to selectively apply load to the region using a hip extension or knee flexion bias without unduly overloading recovering tissues. In the intermediate stages, the clinician can be selective in the application of load to encourage tissue regeneration via morphologic adaptations. During the late stage of rehabilitation, it would seem reasonable to apply both hip- and knee-orientated hamstring strengthening to target all components of the muscular tendinous unit to facilitate full recovery.

The recovering tissues should be loaded using concentric muscle actions initially through the pain-free range of motion. ${ }^{60}$ It is generally accepted that the middle range of motion is the optimal physiologic operating position for muscles via the length-tension relationship. ${ }^{1}$ This midposition in the available range of motion allows optimal force generation in a relatively safe position for the healing tissues. ${ }^{16,38}$ Therefore, we recommend machine-based resistance training to provide a specific overload to the recovering tissues.

It is prudent at this stage of rehabilitation to optimize strength and neuromuscular control through the lumbopelvic region. ${ }^{41}$ This is a transitional region of the body where the hamstrings and its associated musculotendinous components interact with the trunk. Theoretical and observational evidence supports assessment of this region for performance in athletes with hamstring injuries. Maintenance of spinal and lumbopelvic integrity during skilled movements is determined by the muscular capacity of the trunk and its ability to interpret and process sensory input to manage predicable and unpredictable task challenges. During climbing, trunk muscle performance is critical for the transference, absorption, and generation of forces through the lumbopelvic region. ${ }^{65}$ Theoretically, trunk musculature able to tolerate high forces may minimize and load transfer optimally though the hamstring musculature. ${ }^{51}$ The superficial plantar flexor group (gastrocnemius and soleus) should also be considered at this stage because of their functional significance to the heel hook technique. The gastrocnemius and soleus provide powerful plantar flexion torque to increase frictional forces between 
the climber's heel and the rock. Therefore, it seems reasonable to assess and manage strength-related impairments associated with the superficial plantar flexor group.

\section{PHASE 3: HIGH LOAD STRENGTHENING AND FUNCTIONAL RECONDITIONING}

During this phase, the muscular tissues are trained with increasing load and regularity in a resistive protocol. The hamstring musculature should be trained using a concentric muscle action through the entire range of motion. The emphasis in this stage is to ensure that the musculotendinous tissues are exposed to high resistive loads both at the outer range and inner ranges of knee flexion..$^{4,16,29}$ This type of loading encourages a sports-specific transition as the climbing athlete can expect to generate high loads at various points in the range of motion, dependent on the nature of the climbing problem.

At this stage, the exercise modality will favor a unilateral/single-leg approach to resistive loading in keeping with the principle of specific adaptations to imposed demands. ${ }^{59}$ The unilateral loads using modalities such as single-leg knee flexion machines, single-leg stiff leg deadlifts, and single-leg Romanian deadlifts with external tibia rotation will add loading specificity. ${ }^{66,67}$ Rate of force development or explosive training for the hamstring musculature should feature in the rehabilitation program because of its universal application to sports performance. ${ }^{61}$ The rate at which the climber can accelerate the hip over the knee during a rockover technique may be performance enhancing in certain situations (eg, when needing to rockover and latch on to a hold at speed). Often speed of movement is a critical component of performance not achieved by slow strength training. ${ }^{68}$ Therefore, we recommend training to develop strength speed using medicine balls and body weight loads to develop the ballistic qualities of the hamstring muscles. We also recommend specific training of the trunk musculature using both upper and lower body plyometric exercise to manage the absorption of forces through the lumbopelvic complex and to facilitate load transference to the hamstring musculature. ${ }^{65,69}$

\section{RETURN TO CLIMBING}

Currently, there are no return-to-climbing-based criteria for the climbing athlete after an intramuscular tear of the hamstring muscles or injury of the tendon. The evidence base is largely extrapolated from the return-to-sports criteria in sports such as soccer ${ }^{70}$ rugby, and Australian-rules rugby-${ }^{71}$ However, the principles for these sports can be applied judiciously with due regard to the sport-specific nature of climbing. It is clear from experiences in other sports that robust and evidenced-based return-to-sports criteria are sensible based upon the high rate of recurrence associated with

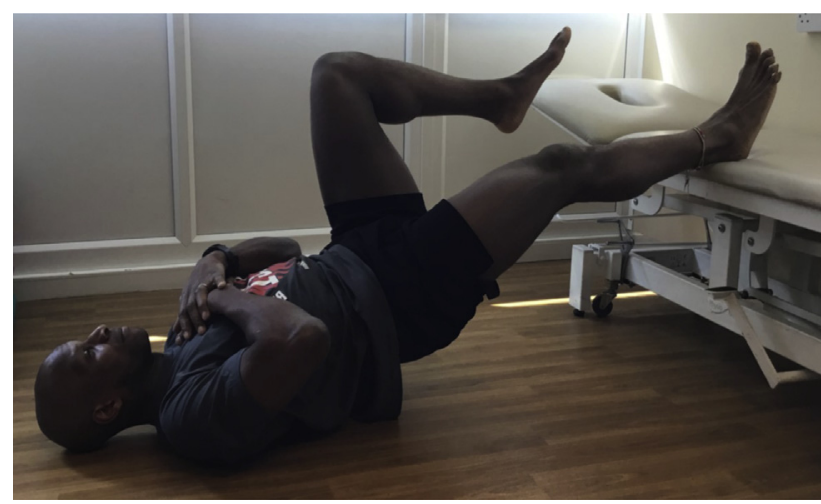

Figure 6. Single leg hamstring bridge.

hamstring injuries. ${ }^{70}$ Indeed, it is often suggested that failure to address the rehabilitation needs of the athlete is a critical factor in the recurrence of these injuries. ${ }^{38,70,72}$ Therefore, we advocate that a pragmatic approach be adopted when considering the suitability of the climber to return to full, unrestricted climbing after a heel hookrelated injury, including the following guidance (Figure 7).

\section{Absence of tenderness on palpation deep to the focal area of injury}

Tenderness in this region may be suggestive of an inappropriate healing response and ability of the tissue to tolerate sports-specific loads. ${ }^{1,73-75}$ However, this should be considered against the background of the chronicity of the disorder and whether this increased tissue sensitivity is related to central sensitization and a secondary hyperalgesia response, which can masquerade as a delayed healing response. The critical factor to assist in differentiating central sensitization, secondary hyperalgesia, and primary hyperalgesia will be the time course of symptoms and stage of biological tissue healing.

\section{Strength testing of the injured tissues within 5\% of the contralateral limb}

Strength testing is an important component for all return-tosports protocols involving musculotendinous tissues. Hamstring muscle testing often involves objective measurement of contractile function using isokinetic dynamometers. ${ }^{71}$ It has been suggested that the peak knee flexion angle should be similar between the injured and uninjured side. ${ }^{70}$ However, this is using isokinetic testing, which is beyond access for most clinicians involved in the rehabilitation of climbers. Therefore, it is recommended that the strength deficit between the injured side and the contralateral side for resisted knee flexion be no more than 5\%, regardless of the modality used to determine the impairment. ${ }^{76}$ Where 


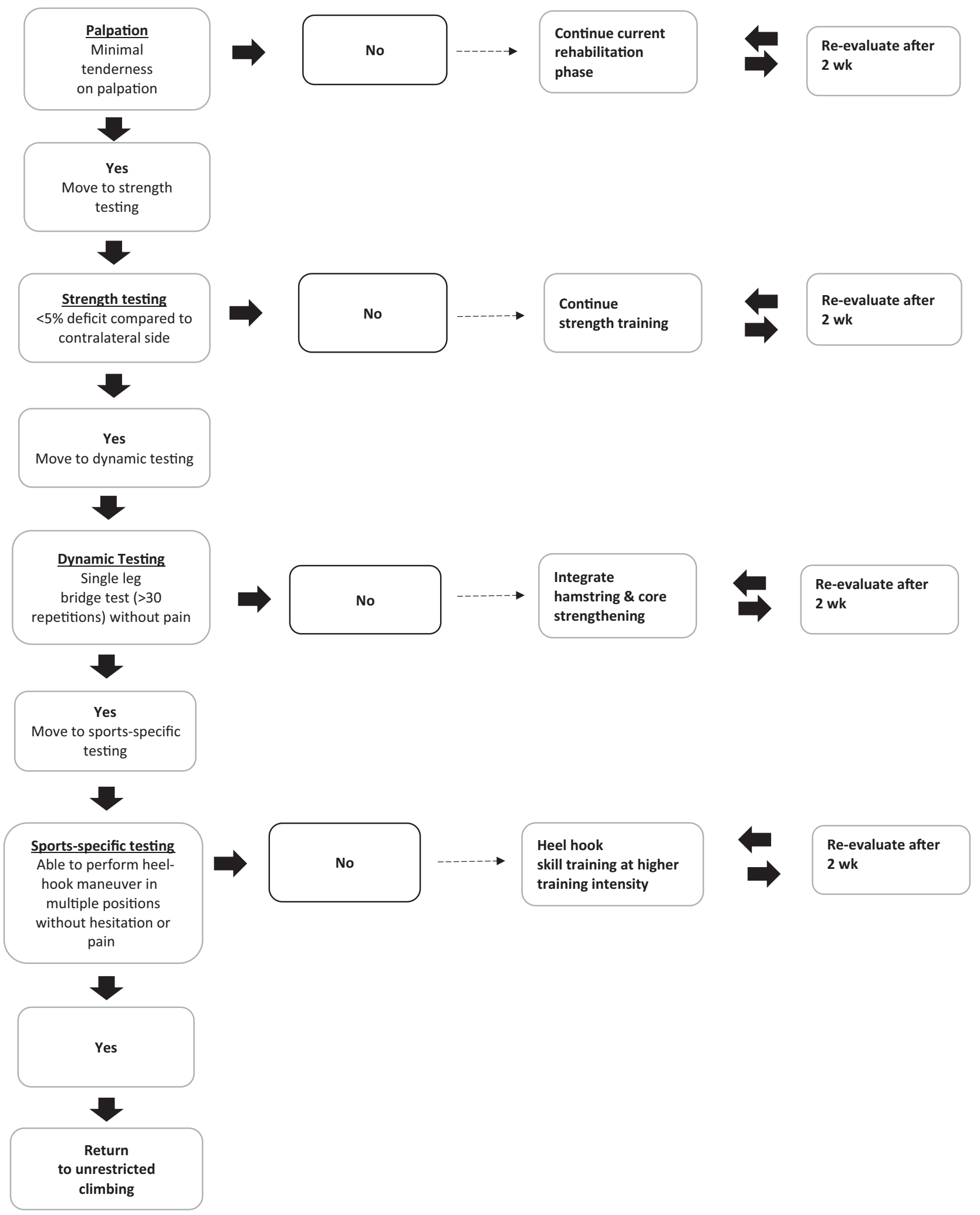

Figure 7. Adapted algorithm for return to climbing. ${ }^{79}$ 
the clinician does not have access to isokinetic testing, we recommend using either resistance training equipment (eg, a knee curl machine in single-leg mode) or isometric strength testing in standardized positions (midrange, inner range, and outer range knee flexion) using a hand-held dynometer. ${ }^{77,78}$

\section{Single-leg hamstring bridge}

This has been recommended as a screening test for strength endurance of the posterior thigh in injured athletes after a hamstring muscle injury (Figure 6). ${ }^{79}$ The test is a field test used in high-performance sports. ${ }^{80}$ It requires in the first instance an appropriate amount of knee flexion force to overcome the inertia of gravity acting on the athlete's body; it therefore is an appropriate test of strength to weight ratio relative to the climber. The climber's hip and lumbopelvic neuromuscular control is tested through the need to establish a hip and pelvic girdle base of support to maintain repeated forceful concentric and eccentric contractions of the hamstring muscles. A score of 20 repetitions or less is considered poor, 25 repetitions is average, and above 30 repetitions is considered good. However, this test has not been validated as a rehabilitation return-to-sports outcome measure. Therefore, we recommend that clinicians use the results of this test to provide a functional indication of hamstring muscle strength endurance within the broader clinical context.

\section{Sports-specific demonstration of the heel hook}

The final test is a sport-specific demonstration of the heel hook while completing a standardized climbing problem with minimal upper body assistance. This is to focus emphasis on the heel hook movement and test the robustness of the athlete. This final test also serves to illustrate to climbing athletes that psychologically they are ready to return to full, unrestricted climbing. ${ }^{81}$

\section{Conclusions}

Rehabilitation of the climbing athlete with a hamstring injury requires an evidence-based approach and professional reasoning on the part of the treating clinician. Rehabilitation should address the loading capacity of injured tissues early in the treatment regime using primarily concentric muscle-strengthening strategies. The clinician must understand the physical demands of bouldering and the kinetic and kinematics associated with the heel hook technique to design an effective rehabilitation and reconditioning program. Return-to-sport testing criteria will provide the climbing athlete and clinician with objective evidence of the athlete's suitability to return to the original injury mechanism and full sports participation.
Acknowledgments: This work is in memory of Ugo Ehiogu and Chief Chike Ehiogu.

Author Contributions: Responsible for concept, design, and drafting of the manuscript (UA); assistance with drafting and revision of the manuscript (VS, GJ, GS); approval of final manuscript (UA, VS, GJ, GS).

Financial/Material Support: None.

Disclosures: None.

\section{References}

1. Heiderscheit BC, Sherry MA, Silder A, Chumanov ES, Thelen DG. Hamstring strain injuries: recommendations for diagnosis, rehabilitation, and injury prevention. J Orthop Sports Phys Ther. 2010;40(2):67-81.

2. Ahmad CS, Redler LH, Ciccotti MG, Maffulli N, Longo UG, Bradley J. Evaluation and management of hamstring injuries. Am J Sports Med. 2013;41(12):2933-47.

3. Valle X, Alentorn-Geli E, Tol JL, Hamilton B, Garrett Jr WE, Pruna R, et al. Muscle injuries in sports: a new evidence-informed and expert consensus-based classification with clinical application. Sports Med. 2017;47(7): $1241-53$.

4. Mueller-Wohlfahrt HW, Haensel L, Mithoefer K, Ekstrand J, English B, McNally S, et al. Terminology and classification of muscle injuries in sport: the Munich consensus statement. Br J Sports Med. 2013;47(6):342-50.

5. Sherry MA, Johnston TS, Heiderscheit BC. Rehabilitation of acute hamstring strain injuries. Clin Sports Med. 2015;34(2): 263-84.

6. Brukner P, Nealon A, Morgan C, Burgess D, Dunn A. Recurrent hamstring muscle injury: applying the limited evidence in the professional football setting with a seven-point programme. Br J Sports Med. 2014;48(11):929-38.

7. Saw R, Finch CF, Samra D, Baquie P, Cardoso T, Hope D, et al. Injuries in Australian rules football: an overview of injury rates, patterns, and mechanisms across all levels of play. Sports Health. 2018;10(3):208-16.

8. O'Sullivan K, O'Ceallaigh B, O'Connell K, Shafat A. The relationship between previous hamstring injury and the concentric isokinetic knee muscle strength of Irish Gaelic footballers. BMC Musculoskelet Disord. 2008;9:30.

9. Bennell KL, Crossley K. Musculoskeletal injuries in track and field: incidence, distribution and risk factors. Aust J Sci Med Sport. 1996;28(3):69-75.

10. Macdonald B, McAleer S, Kelly S, Chakraverty R, Johnston M, Pollock N. Hamstring rehabilitation in elite track and field athletes: applying the British Athletics Muscle Injury Classification in clinical practice. Br J Sports Med. 2019;53(23):1464-73.

11. Kenneally-Dabrowski CJB, Brown NAT, Lai AKM, Perriman D, Spratford W, Serpell BG. Late swing or early stance? A narrative review of hamstring injury mechanisms during high-speed running. Scand J Med Sci Sports. 2019;29(8):1083-91.

12. Chumanov ES, Heiderscheit BC, Thelen DG. Hamstring musculotendon dynamics during stance and swing phases of high-speed running. Med Sci Sports Exerc. 2011;43(3): 525-32. 
13. Schache AG, Dorn TW, Blanch PD, Brown NAT, Pandy MG. Mechanics of the human hamstring muscles during sprinting. Med Sci Sports Exerc. 2012;44(4):647-58.

14. Chumanov ES, Heiderscheit BC, Thelen DG. The effect of speed and influence of individual muscles on hamstring mechanics during the swing phase of sprinting. J Biomech. 2007;40(16):3555-62.

15. LaStayo P, Marcus R, Dibble L, Frajacomo F, Lindstedt S. Eccentric exercise in rehabilitation: safety, feasibility, and application. J Appl Physiol (1985). 2014;116(11):1426-34.

16. Malliaropoulos N, Mendiguchia J, Pehlivanidis $H$, Papadopoulou S, Valle X, Malliaras P, et al. Hamstring exercises for track and field athletes: injury and exercise biomechanics, and possible implications for exercise selection and primary prevention. Br J Sports Med. 2012;46(12):846-51.

17. Schöffl V, Lutter C, Popp D. The "heel hook"-a climbing-specific technique to injure the leg. Wilderness Environ Med. 2016;27(2):294-301.

18. Stępień K, Śmigielski R, Mouton C, Ciszek B, Engelhardt M, Seil R. Anatomy of proximal attachment, course, and innervation of hamstring muscles: a pictorial essay. Knee Surg Sports Traumatol Arthrosc. 2019;27(3):673-84.

19. Kellis E. Intra- and inter-muscular variations in hamstring architecture and mechanics and their implications for injury: a narrative review. Sports Med. 2018;48(10):2271-83.

20. Clanton TO, Coupe KJ. Hamstring strains in athletes: diagnosis and treatment. J Am Acad Orthop Surg. 1998;6(4): 237-48.

21. De Smet AA, Best TM. MR imaging of the distribution and location of acute hamstring injuries in athletes. AJR Am J Roentgenol. 2000;174(2):393-9.

22. Slavotinek JP, Verrall GM, Fon GT. Hamstring injury in athletes: using MR imaging measurements to compare extent of muscle injury with amount of time lost from competition. AJR Am J Roentgenol. 2002;179(6):1621-8.

23. Jones G, Schöffl V, Johnson MI. Incidence, diagnosis, and management of injury in sport climbing and bouldering: a critical review. Curr Sports Med Rep. 2018;17(11):396-401.

24. Lutter C, El-Sheikh Y, Schöffl I, Schöffl V. Sport climbing: medical considerations for this new Olympic discipline. $\mathrm{Br} J$ Sports Med. 2017;51(1):2-3.

25. Fanchini M, Violette F, Impellizzeri FM, Maffiuletti NA. Differences in climbing-specific strength between boulder and lead rock climbers. J Strength Cond Res. 2013;27(2):310-4.

26. Macdonald JH, Callender N. Athletic profile of highly accomplished boulderers. Wilderness Environ Med. 2011;22(2): $140-3$.

27. Giles LV, Rhodes EC, Taunton JE. The physiology of rock climbing. Sports Med. 2006;36(6):529-45.

28. Thompson RN, Hanratty B, Corry IS. "Heel hook" rockclimbing maneuver: a specific pattern of knee injury. Clin J Sport Med. 2011;21(4):365-8.

29. Suchomel TJ, Stone MH. The relationships between hip and knee extensor cross-sectional area, strength, power, and potentiation characteristics. Sports (Basel). 2017;5(3):66.

30. Sharma A, Geovinson SG, Singh Sandhu J. Effects of a nineweek core strengthening exercise program on vertical jump performances and static balance in volleyball players with trunk instability. J Sports Med Phys Fitness. 2012;52(6): $606-15$.

31. Medernach JPJ, Kleinöder H, Lötzerich HHH. Fingerboard in competitive bouldering: training effects on grip strength and endurance. J Strength Cond Res. 2015;29(8): 2286-95.

32. Grant S, Hynes V, Whittaker A, Aitchison T. Anthropometric, strength, endurance and flexibility characteristics of elite and recreational climbers. J Sports Sci. 1996;14(4):301-9.

33. Grant S, Hasler T, Davies C, Aitchison TC, Wilson J, Whittaker A. A comparison of the anthropometric, strength, endurance and flexibility characteristics of female elite and recreational climbers and non-climbers. J Sports Sci. 2001;19(7):499-505.

34. Landin D, Thompson M, Reid M. Knee and ankle joint angles influence the plantarflexion torque of the gastrocnemius. J Clin Med Res. 2015;7(8):602-6.

35. Al Attar WSA, Soomro N, Sinclair PJ, Pappas E, Sanders RH. Effect of injury prevention programs that include the Nordic hamstring exercise on hamstring injury rates in soccer players: a systematic review and meta-analysis. Sports Med. 2017;47(5):907-16.

36. Brooks JHM, Fuller CW, Kemp SPT, Reddin DB. Incidence, risk, and prevention of hamstring muscle injuries in professional rugby union. Am J Sports Med. 2006;34(8): 1297-306.

37. Verrall GM, Slavotinek JP, Barnes PG. The effect of sports specific training on reducing the incidence of hamstring injuries in professional Australian Rules football players. $\mathrm{Br} J$ Sports Med. 2005;39(6):363-8.

38. Bourne MN, Timmins RG, Opar DA, Pizzari T, Ruddy JD, Sims C, et al. An evidence-based framework for strengthening exercises to prevent hamstring injury. Sports Med. 2018;48(2):251-67.

39. Shadle IB, Cacolice PA. Eccentric exercises reduce hamstring strains in elite adult male soccer players: a critically appraised topic. J Sport Rehabil. 2017;26(6):573-7.

40. Mosler AB, Agricola R, Weir A, Hölmich P, Crossley KM. Which factors differentiate athletes with hip/groin pain from those without? A systematic review with meta-analysis. $\mathrm{Br} J$ Sports Med. 2015;49(12):810.

41. Wirth K, Hartmann H, Mickel C, Szilvas E, Keiner M, Sander A. Core stability in athletes: a critical analysis of current guidelines. Sports Med. 2017;47(3):401-4.

42. Paoloni JA, Milne C, Orchard J, Hamilton B. Non-steroidal anti-inflammatory drugs in sports medicine: guidelines for practical but sensible use. Br J Sports Med. 2009;43(11): 863-5.

43. Williams K. Evidence on NSAID use in soft tissue injuries. Nurs Times. 2012;108(45):12-4.

44. van den Bekerom MPJ. No difference in pain, swelling or function with NSAIDs compared with paracetamol for soft tissue injury. Evid Based Nurs. 2016;19(1):21.

45. Whitehead PB. Oral NSAIDs versus other oral analgesic agents for acute soft tissue injury. Int J Evid Based Healthc. 2016;14(3):138-9. 
46. Kouzaki K, Nakazato K, Mizuno M, Yonechi T, Higo Y, Kubo Y, et al. Sciatic nerve conductivity is impaired by hamstring strain injuries. Int J Sports Med. 2017;38(11): 803-8.

47. Bonser RJ, Hancock CL, Hansberger BL, Loutsch RA, Stanford EK, Zeigel AK, et al. Changes in hamstring range of motion after neurodynamic sciatic sliders: a critically appraised topic. J Sport Rehabil. 2017;26(4):311-5.

48. Fyfe JJ, Opar DA, Williams MD, Shield AJ. The role of neuromuscular inhibition in hamstring strain injury recurrence. J Electromyogr Kinesiol. 2013;23(3):523-30.

49. Hughes L, Paton B, Rosenblatt B, Gissane C, Patterson SD. Blood flow restriction training in clinical musculoskeletal rehabilitation: a systematic review and meta-analysis. $\mathrm{Br} \mathrm{J}$ Sports Med. 2017;51(13):1003-11.

50. Yow BG, Tennent DJ, Dowd TC, Loenneke JP, Owens JG. Blood flow restriction training after Achilles tendon rupture. J Foot Ankle Surg. 2018;57(3):635-8.

51. Shield AJ, Bourne MN. Hamstring injury prevention practices in elite sport: evidence for eccentric strength vs. lumbo-pelvic training. Sports Med. 2018;48(3):513-24.

52. Folland JP, Williams AG. The adaptations to strength training: morphological and neurological contributions to increased strength. Sports Med. 2007;37(2):145-68.

53. Schoenfeld BJ, Grgic J, Ogborn D, Krieger JW. Strength and hypertrophy adaptations between low- vs. high-load resistance training: a systematic review and meta-analysis. J Strength Cond Res. 2017;31(12):3508-23.

54. American College of Sports Medicine position stand. Progression models in resistance training for healthy adults. Med Sci Sports Exerc. 2009;41(3):687-708.

55. Tomberlin JP, Basford JR, Schwen EE, Orte PA, Scott SC, Laughman RK, et al. Comparative study of isokinetic eccentric and concentric quadriceps training. J Orthop Sports Phys Ther. 1991;14(1):31-6.

56. Mjølsnes R, Arnason A, Østhagen T, Raastad T, Bahr R. A 10-week randomized trial comparing eccentric vs. concentric hamstring strength training in well-trained soccer players. Scand J Med Sci Sports. 2004;14(5):311-7.

57. Seger JY, Arvidsson B, Thorstensson A. Specific effects of eccentric and concentric training on muscle strength and morphology in humans. Eur J Appl Physiol Occup Physiol. 1998;79(1):49-57.

58. Comfort P, Green C, Matthews M. Training considerations after hamstring injury in athletes. Strength Cond J. 2009;31(1):68-74.

59. Suchomel TJ, Nimphius S, Bellon CR, Stone MH. The importance of muscular strength: training considerations. Sports Med. 2018;48(4):765-85.

60. Brumitt J, Cuddeford T. Current concepts of muscle and tendon adaptation to strength and conditioning. Int J Sports Phys Ther. 2015;10(6):748-59.

61. Cormie P, McGuigan MR, Newton RU. Developing maximal neuromuscular power: part 2 - training considerations for improving maximal power production. Sports Med. 2011;41(2):125-46.
62. Hegyi A, Csala D, Péter A, Finni T, Cronin NJ. High-density electromyography activity in various hamstring exercises. Scand J Med Sci Sports. 2019;29(1):34-43.

63. Opar DA, Williams MD, Timmins RG, Dear NM, Shield AJ. Knee flexor strength and bicep femoris electromyographical activity is lower in previously strained hamstrings. J Electromyogr Kinesiol. 2013;23(3):696-703.

64. Bourne MN, Williams MD, Opar DA, Al Najjar A, Kerr GK, Shield AJ. Impact of exercise selection on hamstring muscle activation. Br J Sports Med. 2017;51(13): 1021-8.

65. Spencer S, Wolf A, Rushton A. Spinal-exercise prescription in sport: classifying physical training and rehabilitation by intention and outcome. J Athl Train. 2016;51(8): 613-28.

66. Schellenberg F, Taylor WR, Lorenzetti S. Towards evidence based strength training: a comparison of muscle forces during deadlifts, goodmornings and split squats. BMC Sports Sci Med Rehabil. 2017;9:13.

67. Schellenberg F, Lindorfer J, List R, Taylor WR, Lorenzetti S. Kinetic and kinematic differences between deadlifts and goodmornings. BMC Sports Sci Med Rehabil. 2013;5(1):27.

68. Gabriel DA, Kamen G, Frost G. Neural adaptations to resistive exercise: mechanisms and recommendations for training practices. Sports Med. 2006;36(2):133-49.

69. Drezner JA. Practical management: hamstring muscle injuries. Clin J Sport Med. 2003;13(1):48-52.

70. Mendiguchia J, Brughelli M. A return-to-sport algorithm for acute hamstring injuries. Phys Ther Sport. 2011;12(1): 2-14.

71. Hickey JT, Timmins RG, Maniar N, Williams MD, Opar DA. Criteria for progressing rehabilitation and determining returnto-play clearance following hamstring strain injury: a systematic review. Sports Med. 2017;47(7):1375-87.

72. Sole G, Milosavljevic S, Nicholson HD, Sullivan SJ. Selective strength loss and decreased muscle activity in hamstring injury. J Orthop Sports Phys Ther. 2011;41(5): 354-63.

73. Ali K, Leland JM. Hamstring strains and tears in the athlete. Clin Sports Med. 2012;31(2):263-72.

74. Askling CM, Nilsson J, Thorstensson A. A new hamstring test to complement the common clinical examination before return to sport after injury. Knee Surg Sports Traumatol Arthrosc. 2010;18(12):1798-803.

75. De Vos RJ, Reurink G, Goudswaard GJ, Moen MH, Weir A, Tol JL. Clinical findings just after return to play predict hamstring re-injury, but baseline MRI findings do not. Br J Sports Med. 2014;48(18):1377-84.

76. Malliaropoulos N, Isinkaye T, Tsitas K, Maffulli N. Reinjury after acute posterior thigh muscle injuries in elite track and field athletes. Am J Sports Med. 2011;39(2): 304-10.

77. McMaster DT, Gill N, Cronin J, McGuigan M. A brief review of strength and ballistic assessment methodologies in sport. Sports Med. 2014;44(5):603-23. 
78. Mentiplay BF, Perraton LG, Bower KJ, Adair B, Pua YH, Williams GP, et al. Assessment of lower limb muscle strength and power using hand-held and fixed dynamometry: a reliability and validity study. PLOS ONE. 2015;10(10), e0140822.

79. Erickson LN, Sherry MA. Rehabilitation and return to sport after hamstring strain injury. J Sport Health Sci. 2017;6(3): 262-70.
80. Freckleton G, Cook J, Pizzari T. The predictive validity of a single leg bridge test for hamstring injuries in Australian rules football players. Br J Sports Med. 2014;48(8): 713-7.

81. Morrison S, Ward P, duManoir GR. Energy system development and load management through the rehabilitation and return to play process. Int J Sports Phys Ther. 2017;12(4): $697-710$ 